\title{
Estimativa da Biomassa Florestal para Fins Energéticos Utilizando Índices de Vegetação e Dados de Campo, Distrito de Mabalane - Moçambique
}

\section{Estimation of Forest Biomass for Energy Purposes Using Vegetation and Field Data Indices, District of Mabalane - Mozambique}

Idolgy Ribeiro dos Santos Mabunda ${ }^{1}$, Laurindo Antonio Guasselli ${ }^{2}$, Eufrásio Joao Sozinho Nhongo ${ }^{3}$ e Benjamim Bandeira $^{4}$

1 Universidade Save (Unisave), Faculdade de Ciências Naturais e Exatas, Xai-Xai, Moçambique. idolgym2001@ yahoo.com.br ORCID: https://orcid.org/0000-0002-1046-965X

2 Universidade Federal do Rio Grande do Sul (UFRGS), Programa de Pós-Graduação em Sensoriamento Remoto, Porto Alegre, RS, Brasil. laurindo.guasselli@ufrgs.br

ORCID: http://orcid.org/0000-0001-8300-846X

3 Universidade Zambeze, Faculdade de Engenharia Ambiental e dos Recursos Naturais, Chimoio, Moçambique.

eufrasionhongo@yahoo.com

ORCID: http://orcid.org/0000-0002-5453-7845

4 Faculdade de Ciências Naturais e Matemática, Maputo. benjband@ hotmail.com

ORCID: https://orcid.org/0000-0002-1076-9759

Resumo: Na África Subsaariana, a intensa exploração de florestas para a extração da lenha e carvão em áreas áridas e semiáridas. O Distrito de Mabalane abastece de lenha e carvão as cidades de Maputo, Matola e Xai-Xai. Entretanto, existe pouco conhecimento sobre a disponibilidade da biomassa nessas áreas. O presente estudo tem como objetivos: analisar a relação entre NDVI derivado da imagem de satélite e a biomassa estimada em campo; modelar a estimava de biomassa em área árida. Os valores do NDVI foram obtidos a partir da imagem de satélite Landsat-8. No campo, quinze parcelas com área de 30 x 30 m foram georeferenciadas e todas as plantas lenhosas vivas com diâmetro à altura do peito (DAP) igual ou maior que $2.5 \mathrm{~cm}$ foram medidas as alturas e DAPs e suas biomassas estimadas a partir de equações alométricas. Os valores de NDVI nos pontos amostrais variaram entre -0.508 e -0.236 , positivamente correlacionados com os valores de biomassa estimados nas 3 equações, que variaram 5,32 e $56,87 t$. $h^{-1}$. A regressão linear entre NDVI e a biomassa no modelo que apresentou melhor resultado, obteve coeficiente de determinação $\mathrm{R}^{2}=$ 0,882. A equação de regressão ajustada a partir de medição indireta da biomassa e índice de vegetação pela diferença normalizada (NDVI), possibilitou a estimativa de biomassa florestal em áreas semiáridas por sensoriamento remoto, com um erro de $36 \%$ na área do presente estudo. O modelo ajustado pode ser suporte para estudos de estimação da biomassa utilizando sensoriamento remoto e os dados de campo em áreas similares.

Palavras-chave: Biomassa. NDVI. Sensoriamento Remoto. Equações alométricas. Mabalane. Semiárido.

\begin{abstract}
In sub-Saharan Africa, the intense exploitation of forests for the extraction of firewood and coal in arid and semi-arid areas. The District of Mabalane supplies the cities of Maputo, Matola and Xai-Xai with firewood and charcoal. However, there is little knowledge about the availability of biomass in these areas. The present study aims to: analyze the relationship between NDVI derived from the satellite image and the biomass estimated in the field; model the estimate of biomass in arid area. NDVI values were obtained from the Landsat- 8 satellite image. In the field, fifteen plots with an area of $30 \times 30 \mathrm{~m}$ were geo-referenced and all live woody plants with a diameter at breast height (DBH) equal to or greater than $2.5 \mathrm{~cm}$ were measured and heights and DBHs and their biomass estimated from allometric equations. The NDVI values at the sampling points varied between -0.508 and -0.236 , positively correlated with the biomass values estimated in the 3 equations, which ranged from 5.32 to $56.87\{$ t.h $\} \wedge\{-1\}$. The linear regression between NDVI and biomass in the model that presented the best result, obtained a coefficient of determination $\mathrm{R} 2=0.882$. The regression equation adjusted from indirect measurement of biomass and vegetation index by normalized difference (NDVI), made it possible to estimate forest biomass in semi-arid areas by remote sensing, with an error of $36 \%$ in the area of the present study. The adjusted model can be used to support biomass estimation studies using remote sensing and field data in similar areas.
\end{abstract}

Keywords: Biomass. NDVI. Remote Sensing. Allometric equations. Mabalane. Semiarid. 


\section{INTRODUÇÃO}

Tradicionalmente os produtos florestais têm sido utilizados para diversas atividades, não se limitando a madeira, forragem e combustível (BROWN, 1997). Na África Subsaariana, em consequência do aumento da população e do crescimento descontrolado das cidades, assiste-se a um aumento da pobreza urbana, com populações fortemente dependentes de fontes de energia baratas, dominadas pela madeira, levando ao esgotamento dos recursos florestais. Nestas regiões do globo é elevado o consumo de combustíveis lenhosos e de resíduos orgânicos, não se prevendo, tão cedo, outra alternativa viável (CHIDUMAYO ; GUMBO, 2010).

Moçambique tem grande potencial florestal, estimado em $70 \%$ do território nacional (MARZOLI, 2008). Apesar deste potencial, o conhecimento da composição florística, estrutura e crescimento destes ecossistemas e da sua variação temporal é ainda muito limitado, o que dificulta a elaboração de planos de manejo que visam ao uso sustentável dos recursos (MAÚSSE, 2013). A elevada procura da biomassa florestal nos centros urbanos nas cidades de Maputo, Matola e Xai-Xai, leva ao aumento da pressão e exploração pouco regrada dos recursos florestais, associado ao crescimento lento da floresta nativa no Distrito de Mabalane.

A província de Gaza é atualmente a principal área de produção de carvão vegetal que abastece Maputo (ATANASSOV et al., 2012; FALCÃO, 2013), e tem o maior número de licenças para a exploração de carvão vegetal no país (LUZ et al., 2015). Os produtores de carvão em Gaza constantemente movem-se para novas áreas de floresta de mopane (Colophospermum mopane), o que aumenta a distância para a cidade de Maputo. O distrito de Mabalane tem a maior produção de carvão licenciado (LUZ et al., 2015).

Este cenário pode levar à escassez dos recursos florestais num futuro próximo, se essa situação prevalecer. Portanto, os estudos voltados a estimativa da biomassa são de grande importância, possibilitam obter informações para um manejo florestal mais adequado e fornecem estimativas da quantidade desses componentes florestais. Entretanto, os estudos florestais têm se concentrado principalmente na produção comercial de madeira, ignorando outros componentes úteis, como lenha (MAMBOWE, 2006).

Estudos de estimativa da biomassa são trabalhosos, demorados e onerosos, e podem ser realizados por métodos diretos e indiretos (WATZLAWICK; KIRCHNER; SANQUETTA, 2009). As medições diretas têm alto grau de precisão, mas envolvem amostragem destrutiva. As medições indiretas são boas alternativas para a medição de biomassa, implicam em menor dano ambiental, menos mão de obra e menor custo financeiro, e podem ser feitas a partir de imagens de satélite e de equações alométricas (LIMA JÚNIOR et al., 2014). A biomassa florestal é toda a biomassa existente na floresta ou apenas na sua fração arbórea. Em se tratando de biomassa de origem vegetal, vem sendo empregado o termo fitomassa (SANQUETTA, 2002). Para Alemdag (1982 apud WENDLING, 1998), biomassa florestal é a quantidade constituída por organismos no ecossistema em termos de massa.

As equações alométricas ou modelos de regressão, são usadas para estimar a biomassa ou volume da árvore acima do solo, baseados em (DAP) e dados de altura. Essas equações são derivadas de valores medidos do peso da árvore relacionados ao DAP e altura das árvores da amostra (KEBEDE; SOROMESSA, 2018). O uso de equações é um método comum e de custo efetivo para estimar a biomassa de espécies de arvores em florestas ou plantações (RAVINDRANATH; OSTWALD, 2008). No entanto, Brown (1997) adverte que, sempre que possível, devem ser desenvolvidas equações locais de biomassa.

Em sensoriamento remoto, as análises e estimativas de biomassa utilizam Índices de Vegetação (IVs) e variáveis extraídas de imagens de satélite, com a vantagem de proporcionar a acessibilidade de informações em locais remotos e de difícil acesso, além de menos onerosos, em termos de trabalhos de campo, quando comparados aos métodos tradicionais (FERRAZ et al., 2013; LIMA et al., 2017; MATASCI et al., 2018; DUARTE et al., 2019). O sensoriamento remoto é amplamente utilizado para estimativa de biomassa, especialmente de florestas boreais e tropicais, e como ferramenta para obter conjuntos de dados espacialmente contínuos sobre biomassa (LU, 2006; NIJLAND et al., 2009; EISFELDER et al., 2011).

O índice de vegetação pela diferença normalizada (NDVI) é um indicador sensível à quantidade de vegetação verde, uma razão entre a reflectância nas regiões do vermelho e infravermelho próximo do espectro eletromagnético, sendo essas duas bandas espectrais selecionadas em razão de serem mais afetadas pela absorção pela clorofila na folhagem da vegetação verde e consequentemente pela densidade dessa vegetação na superfície (MOREIRA, 2011; FRANCISCO et al., 2012; BAYMA; SANO 2015; NHONGO et al., 2017). 
O sensoriamento remoto em regiões semiáridas pode fornecer informações importantes para a gestão sustentável do uso da terra (QI; WALLACE 2002; DECH et al., 2003; KOGAN et al., 2004; KAWAMURA et al., 2005; MUTANGA; RUGEGE, 2006), a avaliação do risco de incêndios (SANNIER et al., 2002; MUTANGA; RUGEGE 2006; VERBESSELT et al., 2006) e de mudanças climáticas (HIRATA et al., 2001; MOLEELE et al., 2001; COLLINS et al., 2009).

Utilizando sensores de baixa resolução espacial Tucker et al. (1985) encontraram boa correlação entre o NDVI e dados da biomassa herbácea no Senegal, mas observaram uma falta de sensibilidade a $250 \mathrm{~kg}$. ha ${ }^{-1}$. Observação não confirmada por Wylie et al. (1991, 1995), que não encontraram aumento visível na dispersão de pontos abaixo de 300-400 kg. ha-1. Hobbs (1995), correlacionaram medidas de biomassa com quatro IVs, descobriram que as relações lineares entre a biomassa e os IVs quebram em níveis de biomassa $>1000 \mathrm{~kg}$ ha. Diouf e Lambin (2001) testaram a relação linear entre os dados da biomassa e o NDVI por um período de 10 anos, encontram bom relacionamento. NDVI foi aplicado com sucesso em estudos posteriores relacionando com os dados de campo (HOLM; CRIDLAND; RODERICK, 2003; WESSELS et al., 2006; BACCINI et al., 2008).

Sensores de média resolução espacial, principalmente da série Landsat, têm sido utilizados para estimativa de biomassa, devido à sua disponibilidade a longo prazo. Franklin e Hiernaux (1991) aplicaram dados TM em um modelo de transferência radiativa para prever o tamanho e a densidade das árvores, utilizando equações alométricas para derivar folhagem e biomassa lenhosa. Para avaliar a escassez da vegetação, Qi e Wallace (2002), usaram NDVI para calcular a cobertura fraccionada verde da vegetação e introduziram um indicador adicional denominado índice de vegetação senescente, e regressão multivariada para relacionar a biomassa do dossel às imagens do Landsat, com forte correlação.

É importante empregar efetivamente técnicas adequadas para extrair variáveis espectrais para modelagem de estimativa de biomassa (LU et al., 2014). As variáveis potenciais das imagens Landsat incluem bandas espectrais individuais, índices de vegetação, imagens transformadas, imagens texturais e imagens fracionárias (LÓPEZ-SERRANO et al., 2016). Embora muitos índices de vegetação tenham sido propostos (BANNARI et al., 1995), dependendo da complexidade da estrutura da floresta, as bandas espectrais, os índices de vegetação e as variáveis de textura podem variar em suas relações com a biomassa (LU et al., 2014).

Sensores espaciais de alta resolução como IKONOS-2 o QuickBird, e sensores aéreos como o HyMap, têm resolução espacial menor que $5 \mathrm{~m}$. São especialmente úteis em estudos detalhados de biomassa de pequenas áreas, e tipos de vegetação com baixa cobertura do solo, como florestas abertas, e permitem a derivação de atributos de uma única árvore (OZDEMIR, 2008). Thenkabail, Jong e Pebesma (2004) estimaram a biomassa e o estoque de carbono em plantações de palmeiras em savanas africanas, os melhores modelos empíricos entre dados de campo e imagens IKONOS foram exponenciais e envolveram a banda do vermelho ou índices normalizados com as bandas do vermelho e do azul, com imagens da estação seca, baseados em NDVI. Addink et al. (2007), utilizaram o sensor HyMap e encontraram um tamanho ótimo de segmentação de objetos e a combinação ideal de bandas para estimativa de biomassa acima do solo e índice de área foliar.

Dados de radar, em particular o SAR, têm sido amplamente utilizados para mapear biomassa, especialmente em áreas de floresta (ISSA et al., 2020). A energia transmitida dos sistemas SAR ativos penetra na vegetação e a quantidade de retroespalhamento depende da estrutura do dossel. O retorno da SAR é influenciado pela constante dielétrica da vegetação, composta por folhas, galhos, troncos e cascas, que representam a biomassa total (EISFELDER, 2011). As frações de volume, distribuição e orientação das partes da vegetação em relação ao vetor de incidência determinam a magnitude e a fase da energia retroespalhada (CRONIN, 2004). Assim, o sinal está relacionado à biomassa da vegetação, influenciado por parâmetros como umidade do solo e rugosidade. Os sistemas de SAR operam em diferentes comprimentos de onda, sensíveis aos diferentes componentes da vegetação (CRONIN, 2004). Para estimativa de biomassa em zonas semiáridas, o uso de sistemas SAR não é tão prevalecente quanto nas áreas florestais (EISFELDER, 2011).

Grande parte dos estudos para estimativa de biomassa baseada em sensoriamento remoto se concentra em florestas. Esforços para a estimativa de biomassa em regiões áridas e semiáridas são importantes, e diferem daquelas usadas em áreas com vegetação mais densa (EISFELDER et al., 2011).

Neste contexto, o presente estudo tem como objetivos:(1) analisar a relação entre o NDVI de florestas 
derivado das imagens de satélite Landsat-8, sensor OLI e a biomassa estimada em campo a partir de equações alométricas; (2) propor um modelo para estimar a biomassa a partir de imagens obtidas por satélite em uma área semiárida.

\section{MATERIAIS E MÉTODOS}

Esta seção apresenta a localização, as características físicas da área de estudo, bem como os procedimentos metodológicos do processamento dos dados para alcançar os objetivos propostos.

\section{1 Área de estudo}

O distrito de Mabalane localiza-se a Sul de Moçambique entre os paralelos $23^{\circ} 00^{\prime}$ e $24^{\circ} 10^{\prime} \mathrm{S}$, e meridianos $32^{\circ} 05^{\prime}$ e $33^{\circ} 04^{\prime}$ E, com uma superfície de $9.107 \mathrm{~km}^{2}$ (Figura 1). O clima é predominantemente semiárido, com uma estação quente e chuvosa, de novembro a março, e outra seca e amena de abril a outubro. A precipitação média anual é inferior a $500 \mathrm{~mm}$ (Figura $1 \mathrm{D}$ ), as temperaturas médias anuais são superiores a $24^{\circ} \mathrm{C}$ e a umidade relativa média anual varia entre os 60 e $65 \%$ (MAE, 2005).

São predominantes as espécies Colophospemummopane (Benth.) J. Léonard. e a Androstachisjohansonii Prain, bem como outras menos predominantes, como TerminaliasericeaBurch. Ex DC., AfzeliaquanzenzisWelw., Strychnosmadagascarensis Poiret, Vangueriainfauta Burch. e Adansoniadigitata L. (INSTITUTO NACIONAL DE INVESTIGAÇÃO AGRÁRIA, 1999). A espécie mais explorada para combustíveis lenhosos (lenha e carvão) é a Colophospemummopane (Figura 2) seguida da Androstachisjohansonii para estacas e para indústria de parquet.

Figura 1 - Localização da área de estudo. A: Mapa de África destacando Moçambique; B: Mapa de Moçambique destacando a Província de Gaza, Distrito de Mabalane e Área de estudo; C: Área de estudo (Landsat-8/OLI, composição Colorida Pseudo Natural Cores, G(4), Sintética verde e Sintética azul de 22 de Junho de 2018); D: Precipitação.

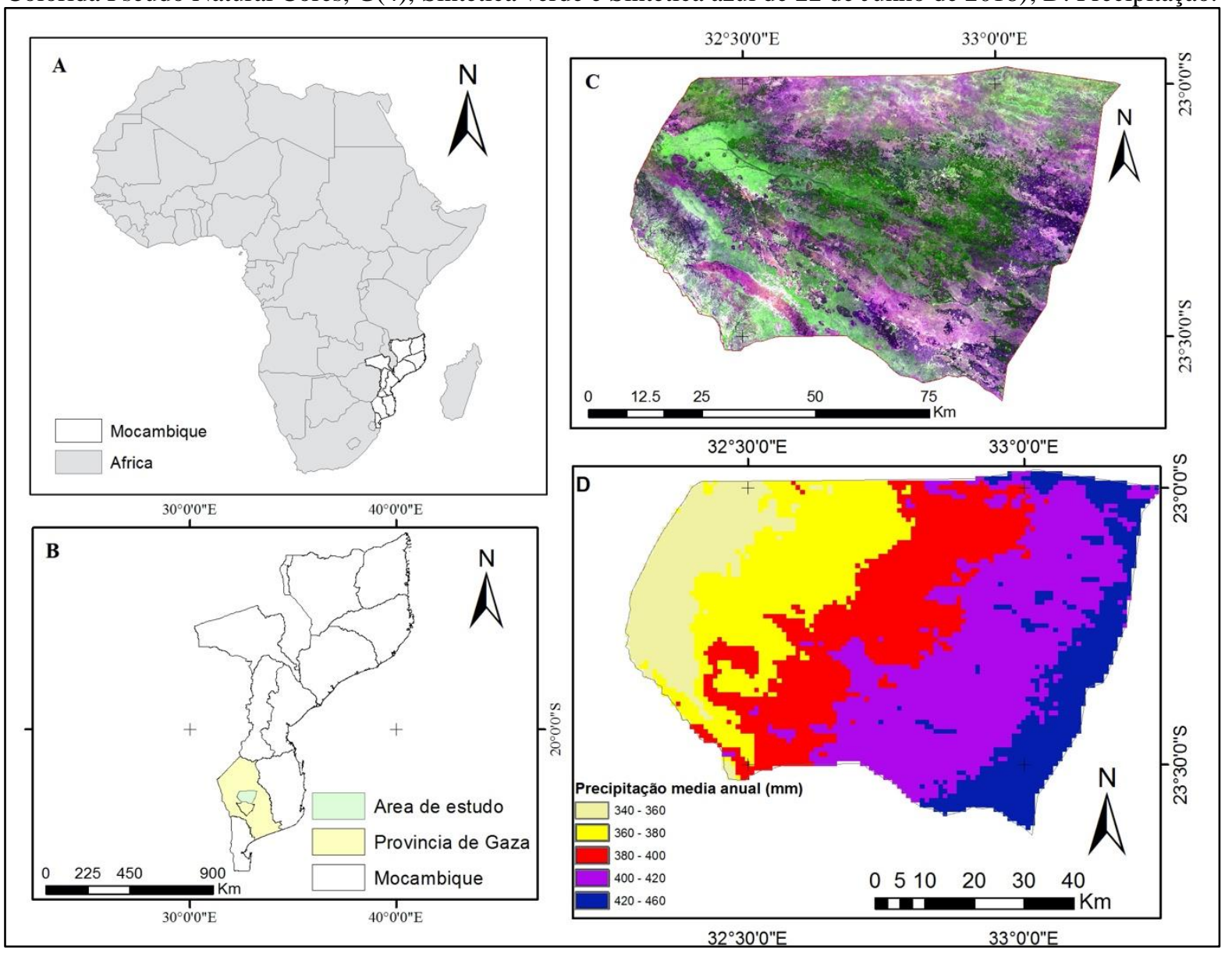

Fonte: Os autores (2021). 
Figura 2 - Espécie de Mopane e carvão vegetal.

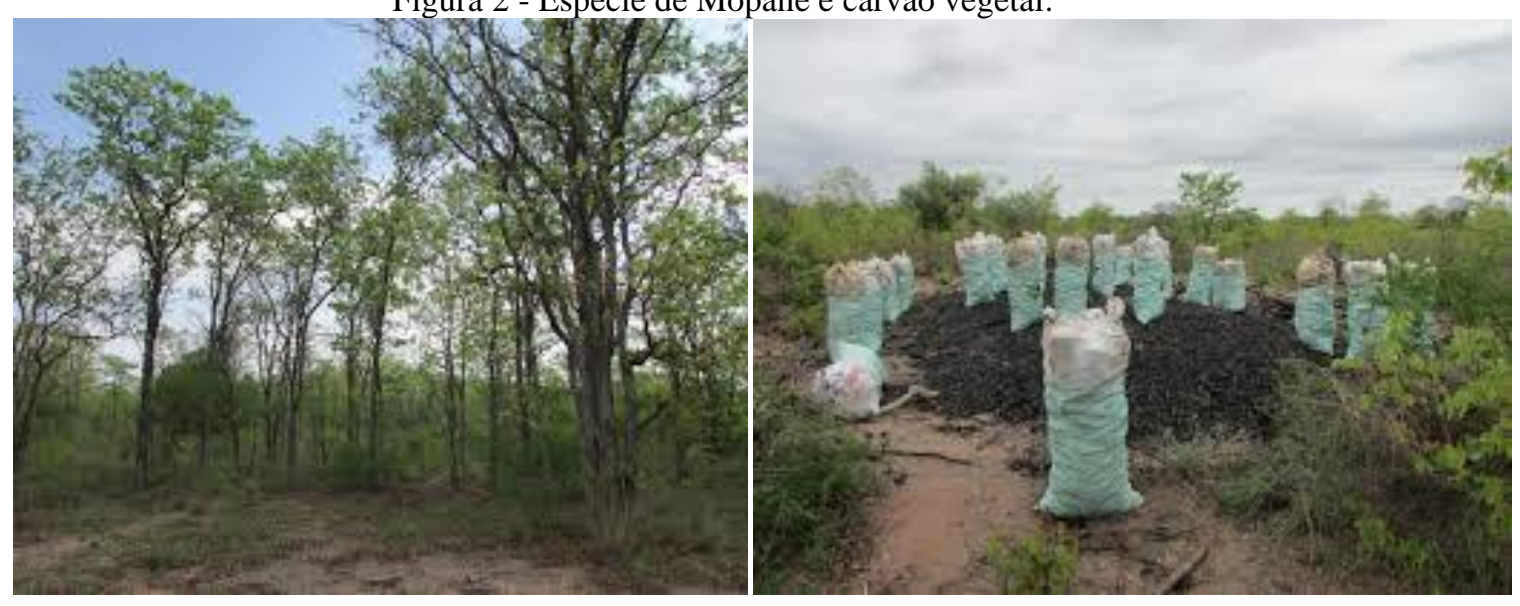

Fonte: Os autores (2021).

\subsection{Determinação do Índice de Vegetação pela Diferença Normalizada (NDVI)}

Foi utilizada uma imagem Landsat-8, sensor OLI (Operational Land Imager), adquirida no dia 22 de Junho de 2018, órbita/ponto 173/55. A imagem foi obtida do site da NASA (National Aeronautics and Space Administration), com Datum WGS 84 (World Geodetic System 1984) e projeção Universal Transversa de Mercator - zona 36S. A imagem foi pré-processada no software QGIS, e realizada a correção radiométrica e a correção atmosférica, pelo método DOS 1 atmospheric correction. O método DOS é um método de correção do espalhamento atmosférico no qual a interferência atmosférica é estimada diretamente a partir dos números digitais (ND) da imagem de satélite, a necessidade de se obter dados sobre as condições atmosféricas na data de obtenção das imagens (SANCHES et al., 2011).

Foi aplicada a equação do Índice de Vegetação por Diferença Normalizada (NDVI), segundo Rouse et al. (1973), Equação 1:

$$
N D V I=\frac{\rho N I R-\rho r e d}{\rho N I R+\rho r e d}
$$

Em que: $\rho N i r=$ bandas do infravermelho próximo; $\rho$ red= banda do vermelho.

Para imagens Landsat 8, as bandas do vermelho e infravermelho próximo são as bandas 4 e 5 (USGS, 2013). O NDVI varia de -1 à 1 , assim quanto mais próximo de 1 maior a densidade da cobertura vegetal, de modo que quanto menor a densidade da vegetação menor o valor do NDVI (PONZONI; SHIMABUKURO, 2007; POLONIO, 2015).

\subsection{Variáveis em campo}

O trabalho de campo foi realizado em Julho de 2019 que coincide com a época seca no hemisfério Sul. Foram definidas um total de 15 parcelas de amostragem de campo, cada uma medindo $30 \mathrm{~m} \times 30 \mathrm{~m}$, e retirada as coordenadas no centro por meio do GPS (Sistema de Posicionamento Global). Nas parcelas foram medidos dados fisionômico-estruturais (DAP e altura). Os indivíduos abordados serão aqueles que apresentarem o DAP $\geq 2.5 \mathrm{~cm}$. É necessário definir um limite inferior do diâmetro sob o qual a madeira não deve ser considerada como lenha (MABOWE, 2006). Portanto, um diâmetro mínimo de 2,5 cm para qualquer componente de madeira de uma árvore ou arbusto parece razoável (DE GIER, 1989).

\subsection{Estimativa da biomassa}

A biomassa foi estimada com uso de equações alométricas. As equações alométricas, são equações de regressão estimada utilizando métodos estatísticos utilizando variáveis de árvores individuais tais como DAP, 
altura, área basal da árvore, volume da árvore (CLARK et al., 2011). A ideia essencial das funções alométricas é estimar variáveis muito difíceis de medir, neste caso a biomassa, através de variáveis de fácil obtenção (tais como as indicadas acima) e sem ter que destruir as árvores. As equações selecionadas foram utilizadas em estudos de áreas que apresentam características similares.

Para a estimativa da biomassa dos componentes da parte aérea foram utilizadas a equações alométricas: Tchauque, 2004 (2); Brown, 1997 (3); e Ryan et al., 2011 (4).

$$
\begin{gathered}
\text { Pstotal }(\mathrm{kg})=-41.077+2.816554 d a p^{2}+0.35657 d a p \\
R^{2}=0,78
\end{gathered}
$$

Em que: Pstotal $(\mathrm{kg})=$ Biomassa total; dap= diâmetro acima do peito; dap ${ }^{2}=$ diâmetro acima do peito ao quadrado; $R^{2}=$ Coeficiente de determinação.

$$
\begin{gathered}
Y(K g)=\exp \{-1.996+2.32 *(D)\} \\
R^{2}=0,89
\end{gathered}
$$

Em que: $Y(\mathrm{~kg})=$ Biomassa total; $D=$ diâmetro acima do peito.

$$
\begin{gathered}
\log (B)=2.601 * \log (d b h)-3.629 \\
R^{2}=0,93
\end{gathered}
$$

Em que: $\log =$ logaritmo natural; $B=$ Biomassa em $\mathrm{kg}$; $d b h=$ diâmetro na altura do peito.

\subsection{Relação entre NDVI e a biomassa estimada}

A relação entre o NDVI e a biomassa estimada pela equação alométrica foi avaliada pela correlação de Pearson. Os dados da biomassa obtidos em campo foram considerados variáveis dependentes e os valores provenientes das imagens sensoriamento remoto, considerados independentes. Os parâmetros da equação do modelo de regressão linear foram estimados pelo método dos mínimos quadrados. Em seguida, foram estimados o coeficiente de correlação de Pearson (R), o coeficiente de determinação $\left(R^{2}\right)$ e a análise de variância. A equação que apresentar melhor ajuste será selecionada.

Para avaliar a precisão do modelo e prever o erro médio das estimativas em trabalhos futuros, foi adotada a validação cruzada deixando um de fora (leave-one-out cross- validation-LOOCV). Este método consiste em, a cada interação criar um modelo com (N-1) dados, considerando $\mathrm{N}$ o número total de dados, que neste caso é igual a 20, denominados dados de treinamento (trainning set), e validar o modelo com o dado que ficou de fora, que é denominado de dado de prova (test set) de fora que, para cada interação, se use um só dado como dado de prova e todos os outros dados são usados como dados de treinamento. Foram realizadas 20 interações, para cada uma foi calculado o erro $E_{i}$. $O$ resultado final foi obtido pela média dos 20 valores de erros obtidos, sendo esta média o erro (E) de predição de valores futuros, de acordo com a Equação 5 (ARLOT; CELISSE, 2010; BERGMEIR; BENÍTEZ, 2012; LIMA JUNIOR et al., 2014, CHEN et al., 2018).

$$
E=\frac{1}{N} \sum_{i=1}^{N} E_{i}
$$

\section{RESULTADOS E DISCUSSÃO}

\subsection{Determinação do NDVI}

Os valores do NDVI foram determinados a partir de uma imagem da estação seca do dia 22 de Junho de 2018 , com variação entre -0.93 e 1 , e nos pontos amostrais entre -0.487 e -0.328 , Figura 3 . Os baixos valores de NDVI nos pontos amostrais relacionam-se a áreas de menor cobertura vegetal relacionadas a baixa 
precipitação. Estes valores são similares aos encontrados em Rishmawi et al. (2016) na resposta da vegetação à variabilidade climática nas zonas áridas do norte a subúmidas da África Subsaariana, em que observaram que a precipitação é o fator que controla o crescimento da vegetação, e a temperatura tem o efeito no crescimento das plantas através das reações químicas (fotossíntese e respiração) que tem o efeito na humidade do solo, entretanto as temperaturas altas podem reduzir a fotossíntese liquida e consequentemente o crescimento da vegetação. Dagnachew et al. (2020), correlacionaram NDVI e fatores climáticos na Etiópia, a fim de determinar o impacto da variabilidade climática na dinâmica da vegetação em escalas temporais e espaciais, e indicaram que a relação entre NDVI e chuva evolui temporalmente e espacialmente. O clima é o fator principal que explica o baixo NDVI na África Subssariana (CAMBERLIN et al., 2007; LI et al., 2015).

Figura 3 - Valores do NDVI da área do estudo, imagens Landsat-8, sensor OLI (Operational Land Imager) de 22 de Junho de 2018.

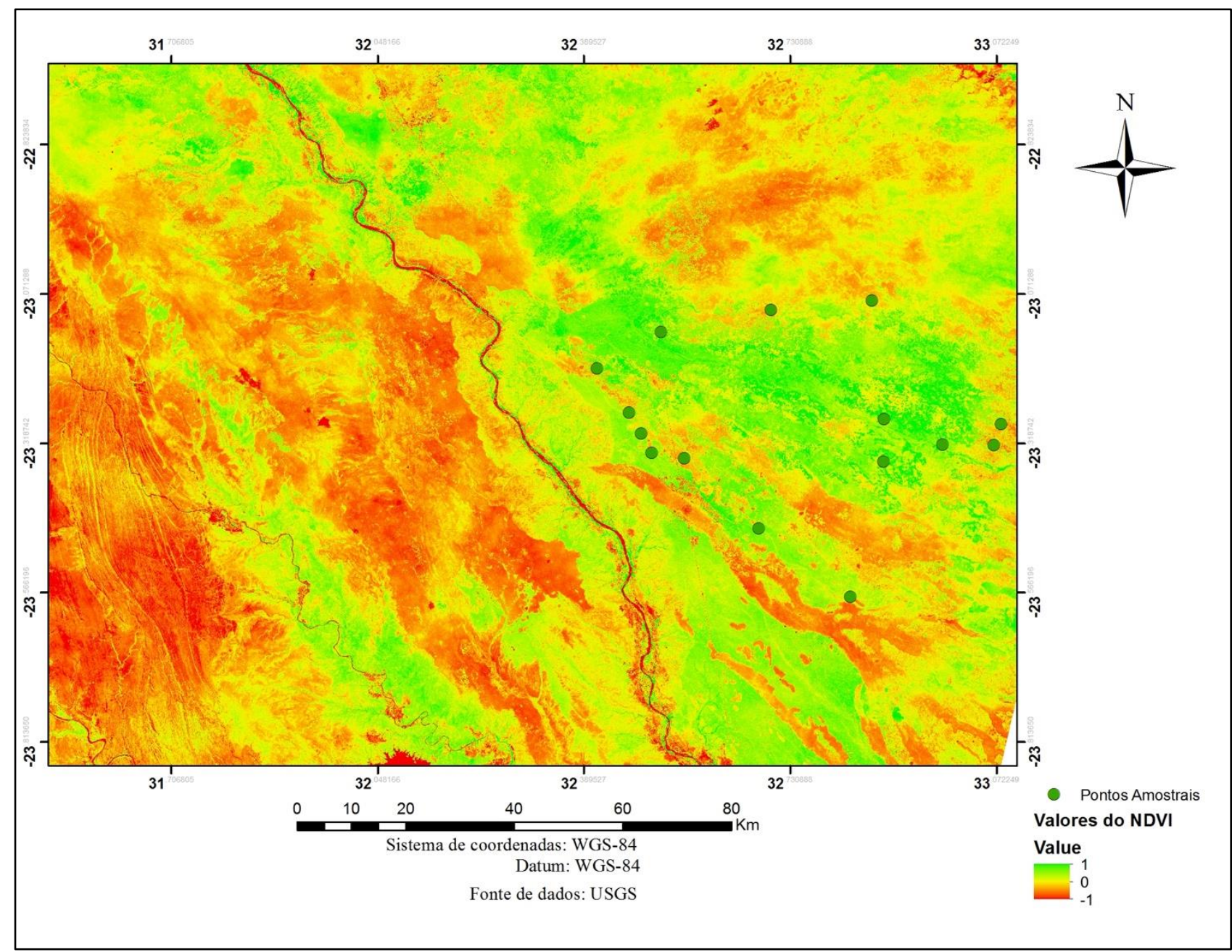

Fonte: Os autores (2021).

\subsection{Estimativa da biomassa}

A estimativa da biomassa, tabela 1, expressa em toneladas por hectare, das áreas amostrais por meio de três equações alométricas. Os resultados das 3 equações variaram entre 5,32 a 56,87 t. $\mathrm{h}^{-1}$. A biomassa estimada por hectare foi inferior a estimada por Wondrade et al. (2015) na Etiópia, através da integração do sensoriamento remoto e equações alométricas de 87,7-308,6 t. $\mathrm{h}^{-1}$ na floresta natural e Amorim, Sampaio, Araújo (2005), 20 a 25 t. h ${ }^{-1}$. Esses resultados encontram-se dentro da amplitude obtida por Carreiras, Melo, Vasconcelos (2013), entre 0,9 e 15,5 t. $\mathrm{h}^{-1}$, na província da Zambezia-Moçambique da biomassa acima do solo nas florestas de savana de Miombo (Moçambique, África Oriental) usando radar de abertura sintética de banda L; por Ribeiro et al. (2008), entre 1,2 e 8,9 t. $\mathrm{h}^{-1}$ na Província de Niassa-Moçambique no mapeamento de biomassa acima do solo e índice de área foliar (LAI) na Reserva do Niassa, norte de Moçambique e Wollen 
et al. (2016) entre 1,6 e 11,8 t. $\mathrm{h}^{-1}$ no Distrito de Mabalane para avaliar os serviços ecossistêmicos de produção de carvão na floresta de Mopane, onde a taxa de crescimento e recuperação das C. mopane é lenta devido a baixa precipitação e más condições do solo.

Koala et al. (2017) advertem que as variáveis obtidas em campo determinam a predição da estimativa da biomassa. De acordo com Abola, Arevalo e Fernandez (2005), as equações alométricas são fortemente diferentes para diferentes espécies de árvores na mesma zona climática, ao utilizar DAP como única variável para estimar a biomassa. Essa variável também foi utilizada por Mate, Johansson e Sitoe (2014), Li, Lin e Yen (2016) e Kebede e Soromessa (2018) que obtiveram resultados satisfatórios. Destaca-se assim, a importância que pesquisas desenvolvam equações específicas a nível local para estimativas mais precisas da biomassa, levando em consideração a zona climática, o tipo de floresta e outros fatores relevantes.

Tabela 1 - Estimativa da biomassa através das 3 equações selecionadas para o estudo.

\begin{tabular}{|c|c|c|c|c|c|}
\hline \multirow[t]{2}{*}{ Amostras } & \multicolumn{2}{|c|}{ Coordenadas } & \multicolumn{3}{|c|}{ Estimativa da biomassa $\left(t \cdot h^{-1}\right)$} \\
\hline & Lat. & Long. & Equação 2 & Equação 3 & Equação 4 \\
\hline 1 & $23^{\circ} 20^{\prime} 3.502^{\prime \prime S}$ & $32^{\circ} 30^{\prime} 6.350^{\prime \prime} \mathrm{E}$ & 48.59 & 30.78 & 47.01 \\
\hline 2 & $23^{\circ} 18^{\prime} 8.24^{\prime \prime S}$ & $32^{\circ} 29^{\prime} 4.150 " \mathrm{E}$ & 32.03 & 26.43 & 35.55 \\
\hline 3 & $23^{\circ} 8 ' 4.301 " \mathrm{~S}$ & $32^{\circ} 31^{\prime} 1.250^{\prime \prime} \mathrm{E}$ & 46.76 & 35.20 & 44.71 \\
\hline 4 & $23^{\circ} 16^{\prime} 43.53 " \mathrm{~S}$ & $32^{\circ} 53^{\prime} 9.49 " \mathrm{E}$ & 56.87 & 37.04 & 49.56 \\
\hline 5 & $23^{\circ} 17^{\prime} 13.67^{\prime \prime S}$ & $33^{\circ} 4^{\prime} 48.25^{\prime \prime} \mathrm{E}$ & 28.12 & 22.07 & 22.22 \\
\hline 6 & $23^{\circ} 19^{\prime} 29.19^{\prime \prime} \mathrm{S}$ & $33^{\circ} 4$ '5.62"E & 5.32 & 21.03 & 21.32 \\
\hline 7 & $23^{\circ} 19^{\prime} 14.34^{\prime \prime S}$ & $32^{\circ} 58^{\prime} 59.68^{\prime \prime} \mathrm{E}$ & 32.76 & 26.30 & 33.56 \\
\hline 8 & $23^{\circ} 20^{\prime} 55.88^{\prime \prime S}$ & $32^{\circ} 53^{\prime} 7.32^{\prime \prime} \mathrm{E}$ & 43.43 & 34.12 & 46.23 \\
\hline 9 & $23^{\circ} 11^{\prime} 41.53^{\prime \prime S}$ & $32^{\circ} 24^{\prime} 39.87^{\prime \prime} \mathrm{E}$ & 43.32 & 34.45 & 47.87 \\
\hline 10 & $23^{\circ} 16^{\prime} 5.98^{\prime \prime S}$ & $32^{\circ} 27^{\prime} 49.20^{\prime \prime} \mathrm{E}$ & 52.98 & 38.90 & 48.65 \\
\hline 11 & $23^{\circ} 20^{\prime} 38.06^{\prime \prime S}$ & $32^{\circ} 33^{\prime} 19.13^{\prime \prime} \mathrm{E}$ & 52.98 & 38.90 & 48.65 \\
\hline 12 & $23^{\circ} 27^{\prime} 34.54^{\prime \prime S}$ & $32^{\circ} 40^{\prime} 44.61 " \mathrm{E}$ & 45.11 & 36.56 & 45.09 \\
\hline 13 & $23^{\circ} 5^{\prime} 51.45^{\prime \prime S}$ & $32^{\circ} 41^{\prime} 56.43^{\prime \prime} \mathrm{E}$ & 54.43 & 38.02 & 50.32 \\
\hline 14 & $23^{\circ} 4^{\prime} 57.24^{\prime \prime S}$ & $32^{\circ} 51^{\prime} 58.78^{\prime \prime} \mathrm{E}$ & 45.03 & 34.76 & 48.43 \\
\hline 15 & $23^{\circ} 34^{\prime} 24.06^{\prime \prime S}$ & $32^{\circ} 49^{\prime} 48.15^{\prime \prime} \mathrm{E}$ & 28.15 & 21.65 & 21.98 \\
\hline
\end{tabular}

Fonte: Os autores (2021).

\subsection{Relação entre NDVI e os dados de biomassa de campo}

Os resultados da análise entre o NDVI, como variável independente, e a estimativa da biomassa por meio das equações alométricas, como variável dependente, através da correlação de Pearson, é apresentado na Figura 4. Como ilustra a Tabela $2 \mathrm{o} \mathrm{R}^{2}$ varia notavelmente entre os modelos. Essa variabilidade pode se dever a problemas de qualidade de dados e outros processos que afetam a biomassa medida como (pastoreio, queimadas) não considerados na regressão linear simples com o NDVI. Segundo Mbow et al. (2013) e Olsen et al. (2015), a variação temporal e espacial na dominância das espécies afeta significativamente a relação entre NDVI e biomassa.

Figura 4 - Relação entre NDVI e a Biomassa estimada por equações alométricas.
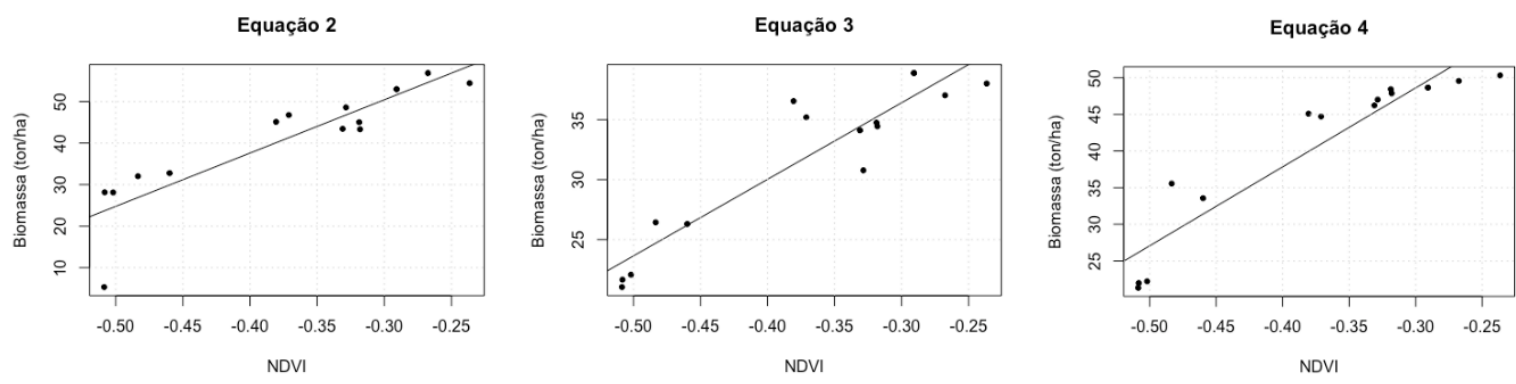

Fonte: Os autores (2021).

Para as equações 2 e 3 a correlação entre a NDVI e a biomassa foi $\left(R^{2}=0.797\right)$ com o valor-p do teste 
$\mathrm{F}=7,54 \cdot 10^{-6}$ e $\left(\mathrm{R}^{2}=0.86\right)$ com o valor-p do teste $\mathrm{F}=5,534 \cdot 10^{-7}$ respectivamente, (Tabela 2). A correlação obtida é semelhante a encontrada por Costa et al. $(2002)$ de $\left(\mathrm{R}^{2}=0,72\right)$ obtida na região do Seridó $(\mathrm{RN})$.

A equação 4 apresentou a melhor correlação entre NDVI e a biomassa $\left(R^{2}=0.88\right)$, com o valor-p do teste $\mathrm{F}=2,095 \cdot 10^{-7}$ este valor é superior à encontrada por Mabowe $(2006) \mathrm{de}\left(\mathrm{R}^{2}=0.024\right)$, ao avaliar a biomassa lenhosa aérea na floresta de Serowe em Botswana. Similar ao obtido por Foody et al. (2001) de (R ${ }^{2}$ $=0.808$ ), para biomassa de florestas tropicais no nordeste de Bornéu, utilizando imagens do Landsat 5TM. Do mesmo modo, Oliveira (2020) ao avaliar o potencial do uso do sensoriamento remoto para estimar as variáveis biofísicas volume, biomassa e carbono em floresta tropical, encontrou uma correlação positiva. Neste caso, Schucknecht et al. (2017) afirmam que o valor de $\mathrm{R}^{2}$ entre a biomassa medida e o NDVI pode ser uma indicação primária da força do relacionamento entre esses dois parâmetros.

Apesar da forca do relacionamento entre os paramentos estimativa de biomassa em regiões semiáridas são problemáticas devido à escassa cobertura vegetal e à vegetação frequentemente senescente (ISSA et al. 2020). A resposta espectral usada para caracterização da vegetação é altamente influenciada pelo fundo do solo, sombra, espécies diferentes, vegetação morta em pé e lixo. Os sinais da vegetação geralmente são muito menores que os do fundo do solo e têm baixo contraste espectral (CALVÃO; PALMEIRIM, 2004).

A produção primária da biomassa total pode ocorrer dentro de um período muito curto; assim, uma alta resolução temporal dos dados de sensoriamento remoto pode ser mais importante que a resolução espacial (TUCKER et al., 1985; SCHUCKNECHT et al., 2017).

As características da vegetação do semiárido, o sensoriamento remoto é utilizado para estimar a biomassa para fins energéticos. A taxa de produção de biomassa determina a quantidade de energia disponível para o uso, a sua estimativa fornece informações cruciais sobre a saúde do ecossistema, por conta da pressão crescente sobretudo na extração e queima da madeira para combustão (HANNN et al., 2008).

Segundo Kumar et al. (2015) são necessárias técnicas que estimem, mapeiem e monitorem com segurança a biomassa em áreas semiáridas. Entender as florestas como fonte de energia renovável, que se forem colocadas sob manejo sustentável, permite conservar a sua cobertura florestal, evitar sua degradação e atender todas as demandas de energia. É necessário também apoiar ações de conservação e manejo, bem como determinar o uso ideal de energia renovável, além de não subestimar a biomassa no balanço energético nacional e regional.

Tabela 2 - Equação desenvolvida da correlação entre NDVI e a biomassa.

\begin{tabular}{c|c|c}
\hline Equação alométrica & $\left(\mathbf{R}^{2}\right)$ & Equação desenvolvida \\
\hline Pstotal $(\mathrm{kg})=-41.077+2.816554 \mathrm{dap}^{2}$ & $\mathrm{R}^{2}=0.797$ & $\mathrm{y}=128.3 \mathrm{x}+88.95$ \\
+0.35657 dap & & $\mathrm{y}=63.90 \mathrm{x}+55.58$ \\
\hline$Y(\mathrm{Kg})=\exp \{-1.996+2.32 *(\mathrm{D})\}$ & $\mathrm{R}^{2}=0.863$ & $\mathrm{y}=107.7 \mathrm{x}+80.94$ \\
\hline $\log (B)=2.601 * \log (\mathrm{dbh})-3.629$ & $\mathrm{R}^{2}=0.88$ & \\
\hline
\end{tabular}

Fonte: Os autores (2021).

A partir dos resultados da relação entre o NDVI como variável independente e a biomassa como variável dependente, selecionou-se a equação que apresentou o melhor resultado na correlação de determinação para o modelo de ajuste tendo resultado na equação 5 com coeficiente de correlação $\mathrm{R}^{2}=0.88$.

$$
\begin{gathered}
t . h \\
(-1)=107.7 N D V I+80.94 \\
\text { Biomassa }
\end{gathered}
$$

A equação resultante foi validada pelo método da validação cruzada (LOOCV), onde o erro de predição foi igual 9,36 t. $\mathrm{h}^{-1}$, equivalente a um erro médio relativo de $36 \%$, considerado satisfatório para um método indireto e para esse tipo de vegetação, que apresenta uma distribuição espacial bastante heterogênea (VIANA; LOPES; ARANHA, 2009; LIMA JUNIOR et al., 2014).

A equação ajustada a partir da correlação entre as equações alométricas e índice de vegetação pela diferença normalizada (NDVI) pode servir como uma solução na estimativa da biomassa utilizando e dados de campo em áreas semiáridas. 


\section{CONCLUSÕES}

Esse estudo verificou uma correlação positiva entre NDVI e a biomassa obtida em campo, calculada por meio das equações alométricas. Todos modelos analisados apresentaram valores de coeficiente de correlação positiva superior a $\left(\mathrm{R}^{2}=0.70\right)$.

A equação de regressão ajustada a partir de medição indireta da biomassa, com equações alométricas e índice de vegetação pela diferença normalizada (NDVI), possibilitou a realização de estimativas de biomassa florestal em áreas de áridas por sensoriamento remoto, com um erro de 36\% na área do presente estudo.

Tendo em conta os dados utilizados (DAP) para a estimativa da biomassa, as áreas amostrais e áreas sem muita vegetação, a qualidade do ajustamento da equação foi razoável. O modelo ajustado pode ser suporte para estudos de estimativa da biomassa utilizando sensoriamento remoto e os dados de campo em áreas similares.

Entretanto, são necessários estudos adicionais que visem aumento de precisão da estimativa, tendo em conta o aumento de números de amostras e mais elementos estruturais da vegetação para o cálculo da biomassa. Sugere-se uso de satélites com múltiplas resoluções espacial e temporal.

Em regiões semiáridas, a estimativa da biomassa visando o conhecimento sobre seu potencial energético e a produção de energia, especialmente a lenha, tem um papel fundamental, porém ainda pouco reconhecido e pouco valorizado, na economia regional.

A elevada procura da biomassa florestal nessas áreas, tem levado ao aumento da pressão e exploração pouco regrada dos recursos florestais. Essa pressão associada ao crescimento lento da floresta nativa tem causado forte impacto e degradação das florestas em Moçambique.

\section{Agradecimentos}

O Deutscher Akademischer Austauschdienst (DAAD), pelo financiamento do doutorado e a Universidade Pedagógica de Moçambique-Gaza, pela concessão da bolsa para estágio no Brasil ao primeiro autor.

\section{Contribuição dos Autores}

Os autores Idolgy Mabunda e Laurindo Guasselli conceberam o projeto. A redação do texto foi feita por Idolgy Mabunda e Laurindo Guasselli. Curadoria dos dados foi feita por Idolgy Mabunda e Eufrásio Nhongo. A revisão e edição do manuscrito foi feita por Idolgy Mabunda, Laurindo Guasselli, Eufrásio Nhongo e Benjamim Bandeira.

\section{Conflitos de Interesse}

Os autores declaram não haver conflitos de interesse.

\section{Referências}

ABOLA, J.; AREVALO, J.; FERNANDEZ, A. Allometric Relationships of Different Tree Species and Stand Aboveground Biomass in the Gomer Laurel Forest (Canary Islands). Flora Morphology, Distribution, Functional Ecology of Plants, v. 200, n. 3, p. 264-274, 2005.

ADDINK, E.A., DE JONG, S.M.; PEBESMA, E.J. Object definition for above- ground biomass and leaf area index estimation. In: 5TH EARSEL WORKSHOP ON IMAGING SPECTROSCOPY, 23-25 APRIL 2007, BRUGES, BELGIUM, 14 pp. Disponível em: <http://5thearselsigis.vgt.vito.be/CD/Fullpapers/Addink_final.pdf>. Acessado em: 25 Nov. 2019.

AMORIM, I. L.; SAMPAIO, E. V. S. B.; ARAUJO, E. L. Flora e estrutura da vegetação arbustivo-arbórea de uma área de catinga do Seridó, RN, Brasil. Acta Botânica Brasilica, São Paulo, v. 19, n. 3, pp. 615-623, 
2005.

ARLOT, S.; CELISSE, A.A survey of cross-validation procedures for model selection. Statistics Surveys, v. 4, p. 40-79, 2010.

ATANASSOV, B.; EGAS, A.; FALCÃO, M.; FERNANDES, F.; MAHUMANE, G. Mozambique urban biomass energy analysis. Relatório técnico, Ministério de energia, p. 56, 2012. Disponível em: <http://greenlight-africa.com/assets/final_report_mozamique_urban_biomass.pdf>. Acesso em: 04 Fev. 2020 .

BACCINI, A., LAPORTE, N.T., GOETZ, S.J., SUN, M.; HUANG, D., A first map of tropical Africa's aboveground biomass derived from satellite imagery. Environmental Research Letters, vol. 3, p. 9, 2008.

BANNARI, A.; MORIN, D.; BONN, F.; HUETE, A.R. A Review of Vegetation Indices. Remote Sens. Rev., vol. 13, p. 95-120, 1995.

BAYMA, A.P.; SANO, E.E. Séries temporais de índices de vegetação (NDVI e EVI) do sensor MODIS para detecção de desmatamentos no bioma Cerrado, Boletim de Ciências Geodésicas, v.21, n.4, pp.797-813, 2015.

BERGMEIR, C.; BENÍTEZ, J. M. On the use of cross- validation for time series predictor evaluation. Information Sciences, v. 191, p. 192-213, 2012.

BILA, J. M. et al. Crescimento e fitossociologia de uma floresta com Colophospermummopane, em Mabalane, Província de Gaza, Moçambique. Pesquisa Florestal Brasileira, vol. 32, n. 72, pp. 421-427, 2002.

BROWN, S. Estimating Biomass and Biomass Change of Tropical Forests: a Primer. FAO Forestry Paper. FAO, Rome, 1997.

CALVÃO, T.; PALMEIRIM, J.M. Mapping Mediterranean scrub with satellite imagery: biomass estimation and spectral behaviour. International Journal of Remote Sensing, n. 25, p. 3113-3126, 2004.

CAMBERLIN, P.; MARTINY, N.; PHILIPPON, N.; RICHARD, Y. Determinants of the inter annual relationships between remote sensed photosynthetic activity and rain-fall in tropical Africa. Remote Sensing of Environment, v. 106. p. 199-216, 2007.

CARREIRAS, J. M. B.; MELO, J. B.; VASCONCELOS, M. J. Estimating the Above-Ground Biomass in Miombo Savanna Woodlands (Mozambique, East Africa) Using L-Band Synthetic Aperture Radar Data. Remote Sensing. n. 5. p. 1524-1548, 2013.

CHEN, W.; ZHAO, J.; CAO, C.; TIAN, H. Shrub biomass estimation in semi-arid sandland ecosystem based on remote sensing technology. Global Ecology and Ecology, v. 16, p. 1-9, 2018.

CHIDUMAYO, E. e GUMBO, D.J. The dry forests and woodlands of Africa: managing for products and services. Eathscan Journal, 2010. Diponivel em:

<https://www.researchgate.net/publication/285906452_The_dry_forests_and_woodlands_of_Africa_ma naging_for_products_and_services/citation/download>. Acessado em: 9 Jun. 2019.

COLLINS, J.N.; HUTLEY, L.B.; WILLIAMS, R.J.; BOGGS, G.; BELL, D.; BARTOLO, R., Estimating landscape-scale vegetation carbon stocks using airborne multi-frequency polarimetric synthetic aperture radar (SAR) in the savannahs of north Australia. International Journal of Remote Sensing, n. 30, pp. 1141-1159, 2009.

COSTA, T. C. E. C.; ACCIOLY, L. J. O.; OLIVEIRA, M. A. J.; BURGOS, N.; SILVA, F. H. B. Phytomass mapping of the Seridó Caatinga vegetation by the plant area and the normalized difference vegetation indices. ScientiaAgricola, Piracicaba, v. 59, n. 4, p. 707-715, 2002.

ClARK, D.A.; BROWN, S.; KICKLIGHTER, D.; CHAMBERS, J.Q.; THOMLINSON, J.R.; NI, J. Measuring net primary production in forests: Concepts and field methods. Ecol. Appl., n. 11, p. 356-370, 2001.

CRONIN, N.L.R., The potential of airborne polarimetric synthetic aperture radar data for quantifying and mapping the biomass and structural diversity of woodlands in semi- arid Australia. $517 \mathrm{f}$. Tese de Doutorado (Biology), University of New South Wales, Sydney, NSW, Australia, 2004. 
DAGNACHEW, M.; KEBEDE, A.; MOGES, A.; ABEBE, A. Effects of Climate Variability on Normalized Difference Vegetation Index (NDVI) in the Gojeb River Catchment, Omo-Gibe Basin, Ethiopia. Advances in Meteorology, v. 13, p. 16, 2020.

DE GIER, A., Woody biomass for fuel - estimating the supply in natural woodlands and shrubslands. Tese (Doutorado em Sistema de Informação Geográfica) ITC, Enschede, The Netherlands. 1989.

DECH, S., BACHMANN, M. e MÜLLER, A. New remote sensing approaches in support of sustainable land use management in semi-arid environments. In: 30th INTERNATIONAL SYMPOSIUM ON REMOTE SENSING OF ENVIRONMENT, 10-14 November 2003, Honolulu, HI, USA. 2003.

DIOUF, A. and LAMBIN, E., Monitoring land-cover changes in semi-arid regions: remote sensing data and field observations in the Ferlo, Senegal. Journal of Arid Environments, vol. 48, p. 129-148, 2001.

DUARTE, P.; AlCON, S. M.; TENA, A. G.; AlGEET, N.; TOMÉ, J. L.; RECUERO, L.; ORUETA, A. P. OYONARTE, C. Improving Aboveground Forest Biomass Maps: From High-Resolution to National Scale. Remote sensing, vol. 11, n. 7, p. 795, 2019.

EISFELDER, C.; KUENZER, C.; DECH, S. Derivation of biomass information for semi-arid areas using remote-sensing data. Remote sensing, vol. 33, p. 1-48, 2011.

FALCÃO MPMP. Produção e Consumo Doméstico de Combustíveis Lenhosos em Moçambique. Universidade Nova de Lisboa; 2013.

FERRAZ, A. S.; SOARES, V. P.; SOARES. C. P. B.; RIBEIRO, C. A. S. A; GLERIANI, J. M. Uso de imagens do satélite IKONOS II para estimar biomassa aérea de um fragmento de floresta estacional semidecidual. Anais do XVI Simpósio Brasileiro de Sensoriamento Remoto - SBSR, Foz do Iguaçú, PR, Brasil, 2013, INPE. Disponível em: <http://www.dsr.inpe.br/sbsr2013/files/p0302.pdf>. Acesso em: 23 de Dez. de 2019.

FOODY, G.M.; CUTLER, M.E.; MCMORROW, J.; PELZ, D.; TANGKI, H.; BOYD, D.S. et al. Mapping the biomass of Bornean tropical rain forest from remotely sensed data, Global Ecology \&Biogeograph, v. 10, p. 379-387, 2001.

FRANCISCO, P. R. M.; CHAVES, I. B.; LIMA, E. R. V.; BANDEIRA, M. M.; SILVA, B. B. Mapeamento da Caatinga com uso de geotecnologia e análise da unidade antecedente em bacia hidrográfica. Revista Brasileira de Geografia Física, vol. 5, n. 3, p. 676-693, 2012.

FRANKLIN, J.; HIERNAUX, P.H.Y., Estimating foliage and woody biomass in Sahelian and Sudanian woodlands using a remote sensing model. International Journal of Remote Sensing, vol. 12, pp. 13871404, 1991.

HANAN, N.P.; SEA, W.B.; DANGELMAYR, G. e GOVENDER, N. Do fires in savannas consume woody biomass? A comment on approaches to modeling savanna dynamics. Am. Nat., v. 171, n. 6, p. 851 -856, 2008. DOI.: 10.1086/523971 AMNTA4 0003-0147.

HIRATA, M., KOGAB, N., SHINJO, H., FUJTTA, H., GINTZBURGER, G. e MIYAZAKI, A., Vegetation classification by satellite image processing in a dry area of northeastern Syria. International Journal of Remote Sensing, vol. 23, p. 507-516, 2001.

HOBBS, T.J. The use of NOAA-AVHRR NDVI data to assess herbage production in the arid rangelands of Central Australia. International Journal of Remote Sensing, n. 16, p. 1289-1302, 1995.

INSTITUTO NACIONAL DE INVESTIGAÇÃO AGRÁRIA. Inventário dos recursos de terra na comunidade de Gerés, Distrito de Mabalane. Série terra e água, p. 77,1999.

ISSA, S.; DAHY, B.; KSIKSI, T.; E SALEOUS, N. A Review of Terrestrial Carbon Assessment Methods Using Geo-Spatial Technologies with Emphasis on Arid Lands. Remote Sens., vol. 12, 2020. DOI.: 10.3390/rs12122008.

KAWAMURA, K., AKIYAMA, T., YOKOTA, H., TSUTSUMI, M., YASUDA, T., WATANABE, O. and WANG, S.P. Quantifying grazing intensities using geographic information sys- tems and satellite remote sensing in the Xilingol steppe region, Inner Mongolia, China. Agriculture, Ecosystems and 
Environment, vol. 107, p. 83-93, 2005.

KEBEDE, B.; SOROMESSA, T. AllometricequationsforabovegroundbiomassestimationofOleaeuropaea L. subsp. cuspidata in Mana Angetu Forest. Ecosystem Health and Sustainability, vol. 4, n. 1, p. 1-12, 2018. DOI.: 10.1080/20964129.2018.1433951.

KOALA J., SAWADOGO L., SAVADOGO P., AYNEKULU E., HEISKANEN J., SAÏD M. Allometric equations for below-ground biomass of four key woody species in West African savanna-wood- lands. Silva Fennica, vol. 51, no. 3, 15 p., 2017. DOI: https.: 10.14214/sf.1631.

KOGAN, F., STARK, R., GITELSON, A., JARGALSAIKHAN, L., DUGRAJAV, C. e TSOOJ, S., Derivation of pasture biomass in Mongolia from AVHRR-based vegetation health indices. International Journal of Remote Sensing, vol. 25, pp. 2889-2896, 2004.

KUMAR, L.; SINHA, P.; TAYLOR, S. e ALQURASHI, A. F. Review of the use of remote sensing for biomass estimation to support renewable energy generation. Journal of Applied Remote Sensing, v. 9, pp. 29, 2015.

LI, S.; YANG, S.; LIU, X.; LIU, Y.; SHI, M. NDVI-Based Analysis on the Influence of Climate Change and Human Activities on Vegetation Restoration in the Shaanxi-Gansu-Ningxia Region, Central China. Remote Sensing, v. 7, n. 9, p. 11163-11182, 2015.

LI, L. E.; LIN, Y. J.; YEN, T. M. Using Allometric Models to Predict the Aboveground Biomass of Thorny Bamboo (Bambusa stenostachya) and Estimate Its Carbon Storage. Taiwan. Journal of Forest Science, v. 31, n. 1, p. 37-47, 2016.

LIMA JÚNIOR, C. de, ACCIOLY, L., GIONGO, V.; LIMA, R. L. F. de A; SAMPAIO, E. V. de Sá B.; MENEZES, R. S. C. Estimativa de biomassa lenhosa da caatinga com uso de equações alométricas e índice de vegeta ção. Sci. For., Piracicaba, v. 42, n. 102, p. 289-298, 2014.

LIMA, D. R. M.; DLUGOSZ, F. L.; IURK, M. C. e PESCK, V. A. Uso de NDVI e SAVI para Caracterização da Cobertura da Terra e Análise Temporal em Imagens RapidEye. Espacios, vol. 38, n. 36, p. 7, 2017.

LÓPEZ-SERRANO, P.M.; CORRAL-RIVAS, J.J.; DÍAZ-VARELA, R.A.; ÁLVAREZ-GONZÁLEZ, J.G.; LÓPEZ-SÁNCHEZ, C.A. Evaluation of radiometric and atmospheric correction algorithms for aboveground forest biomass estimation using Landsat 5 tm data. Remote Sens., vol. 8, p. 369, 2016.

LU, D. The potential and challenge of remote sensing-based biomass estimation. International Journal of Remote Sensing, n. 27, p. 1297-1328, 2006.

LU, D.; CHEN, Q.; WANG, G.; LIU, L.; LI, G.; MORAN, E. A survey of remote sensing based aboveground biomass estimation methods in forest ecosystems. Int. J. Digit. Earth, v. 9, p. 63-105, 2014.

LUZ, A.C. et al. Charcoal production and trade in southern Mozambique: historical trends and present scenarios. In: XIV Forestry Congress XIV, Durban, 2015. Disponível: <https://www.researchgate.net/publication/282253033_Charcoal_production_and_trade_in_southern_M ozambique_historical_trends_and_present_scenarios>. Acesso em: 05 de Fev. 2020.

MABOWE, B. R. Aboveground woody biomass assessment in Serowe woodlands, Botswana. Desertion (degree of Master of Science in Geo-information Science and Earth Observation, Specialisation: Environmental Systems Analysis and Management). International institute for geo-information science and earth observation Enschede, the Netherlands, 2006.

MATASCI, G.; HERMOSILLA, T.; WULDER, M.A.; WHITE, J.C.; COOPS, N.C.; HOBART, G.W.; ZALD, H.S. Large-area mapping of Canadian boreal forest cover, height, biomass and other structural attributes using Landsat composites and lidar plots. Remote Sens. Environ. n. 209, p. 90-106, 2018.

MINISTÉRIO DE ADMINISTRAÇÃO ESTATAL (MAE). Perfil do Distrito de Mabalane Província de Gaza. Ministry of Energy. p. 45, 2005.

MARZOLI, A. Avaliação Integrada de Florestas de Moçambique - Inventário florestal nacional. Relatório final. Direcção Nacional de Terras e Florestas. Ministério da Agricultura. Maputo, Mozambique. 2008

MATE, R.; JOHANSSON, T.; SITOE, A. Biomass equations for Tropical Forest Tree Species in Mozambique. 
Forests, vol. 5, p. 535-556, 2014. DOI.: 10.3390/f5030535.

MAÚSSE, A. Arranjos institucionais na utilização sustentável dos Recursos florestais: Caso da exploração do carvão vegetal nos povoados de Mabomo e Mungaze no Distrito de Mabalane, Gaza, Maputo, 2013.

MBOW, C.; FENSHOLT, R.; RASMUSSEN, K.; DIOP, D. Can vegetation productivity be derived from greenness in a semi-arid environment? Evidence from ground-based measurements. J. Arid. Environ, v. 97, p. 56-65, 2013.

MOLEELE, N., RINGROSE, S., ARNBERG, W., LUNDEN, B. and VANDERPOST, C., Assessment of vegetation indexes useful for browse (forage) prediction in semi-arid rangelands. International Journal of Remote Sensing, vol. 22, p. 741-756, 2001.

MOREIRA, A.; KUPLICH, T. M.; FONSECA, E. L. A variação fenológica de tipologias campestres do bioma Pampa pela abordagem ondaleta. In: XV SIMPÓSIO BRASILEIRO DE SENSORIAMENTO REMOTO, 2011, Curitiba. Anais...Curitiba, INPE, 2011, p.1584.

MUTANGA, O. e RUGEGE, D., Integrating remote sensing and spatial statistics to model herbaceous biomass distribution in a tropical savanna. International Journal of Remote Sensing, vol. 27, p. 3499-3514, 2006.

NHONGO, E.J.S.; FONTANA, D.C.; GUASSELLI, L.A.; ESQUERDO, J.C.D.M. Caracterização fenológica da cobertura vegetal com base em série temporal NDVI/MODIS na reserva do Niassa - Moçambique, Revista Brasileira de Cartografia, vol. 69, n. 6, p. 1175-1187. 2017.

NIJLAND, W.; ADDINK, E.A.; DE JONG, S.M; VAN DER MEER, F.D. Optimizing spa- tial image support for quantitative mapping of natural vegetation. Remote Sensing of Environment, n. 113, p. 771-780, 2009.

OLIVEIRA, G.F.S. Uso de Técnicas de Sensoriamento remoto para estimar variáveis biofísicas em floresta tropical seca, município de Floresta. Dissertação (Mestrado em Ciencias Florestais), Universidade Federal Rural de Pernambuco, Programa de Pós-Graduação em Ciências Florestais, Recife, 2020 .

OZDEMIR, I. Estimating stem volume by tree crown area and tree shadow area extracted from pan-sharpened Quickbird imagery in open Crimean juniper forests. International Journal of Remote Sensing, v. 19, p. 5643-5655, 2008.

OLSEN, J.L.; MIEHE, S.; CECCATO, P.; FENSHOLT, R. Does EO NDVI seasonal metrics capture variations in species composition and biomass due to grazing in semi-arid grassland savannas? Biogeosciences, $\mathrm{v}$. 12, p. 4407-4410, 2015.

POLONIO, V. D. Índices de vegetação na mensuração do estoque de carbono em áreas com cana-deaçúcar. Dissertação (Mestrado em Agronomia: Energia na Agricultura). Universidade Estadual paulista, Faculdade de Ciências Agronômicas de Botucatu, p. 73. 2015.

PONZONI, F. J.; SHIMABUKURO, Y. E. Sensoriamento remoto no estudo da vegetação. $1^{\text {a }}$ ed. São José, dos Campos: Parêntese Editora, 2007.

QI, J.; WALLACE, O. Biophysical attributes estimation from satellite images in arid regions. In: PROCEEDINGS OF THE INTERNATIONAL GEOSCIENCE AND REMOTE SENSING SYMPOSIUM, 2002, Toronto, IGARSS, vol. 4, 2002. p. 2000.

RAVINDRANATH, N. H.; OSTWALD, M. Carbon Inventory Methods: Handbook for Greenhouse Gas Inventory. Carbon Mitigation and Round Wood Production Projects Springer Science. Delft: Advancesin Global Change Research, Springer, 2008.

RIBEIRO, N.; SAATCHI, S.; SHUGART, H.; WASHINGTON-ALLEN, R. Aboveground biomass and leaf area index (LAI) mapping for Niassa Reserve, northern Mozambique. Journal of Geophysical Research, v. 13, 2008. DOI. 10.1029/2007JG000550.

RISHMAWI, K.; PRINCE, S. D.; XUE, Y. Vegetation Responses to Climate Variability in the Northern Arid to Sub-Humid Zones of Sub-Saharan Africa. Remote sensing, vol. 8, p. 910, 2016. 
ROUSE, J. W.; HASS JR., R. H.; SCHELL, J. A.; DERRING, D.W. Monitoring the vernal advancement and retrogradation (green wave effect) of natural vegetation. Prog., Remote Sensing Center, Texas A\&M Univ., College Station, p. 93. 1973.

RYAN, C.M.; WILLIAMS, M.; GRACE, J. Above- and belowground carbon stocks in a Miombo Woodland landscape of Mozambique. Biotropica, v. 43, p. 423-432, 2011.

SANCHES, I.; ANDRADE, R. G.; QUARTAROLI, C. F. e RODRIGUES, C. A. P. Análise comparativa de três métodos de correção atmosférica de imagens Landsat5-TM para obtenção de reflectância de superfície e NDVI. In: XV SIMPÓSIO BRASILEIRO DE SENSORIAMENTO REMOTO, 2011, Curibiba, Anais... Curitiba: INPE, p. 7564, 2011.

SANNIER, C.A.D., TAYLOR, J.C. e PLESSIS, W.D., Real-time monitoring of vegetation biomass with NOAA-AVHRR in Etosha National Park, Namibia, for fire risk assessment. International Journal of Remote Sensing, vol. 23, p. 71-89, 2002.

SANQUETTA, C. R. Métodos de determinação de biomassa florestal. In: SANQUETTA, C. R.; WATZLAWICK, L. F.; BALBINOT, R.; ZILIOTTO, M. A. B.; GOMES, F. dos S. (Eds.). As florestas e o carbono. Curitiba, Embrapa Florestas, 2002. p. 119-140.

SCHUCKNECHT, A.; MERONI, M., KAYITAKIRE, F. E BOUREIMA, A. Phenology-Based Biomass Estimation to Support Rangeland Management in Semi-Arid Environments. Remote Sens., v. 9, n. 463, 2017. DOI:10.3390/rs9050463.

SITOE, A.; TCHAUQUE, F. Medição da biomassa florestal utilizando informação do inventário florestal. Relatório final. Unidade de Inventário Florestal. Direcção Nacional de Terras e Florestas. Maputo, 2007.

TCHAÚQUE, F. D. D. L. J. Avaliação da Biomassa Lenhosa Área no Corredor da Beira, Maputo, Moçambique, p.49, 2004.

THENKABAIL, P.S., STUCKY, N., GRISCOM, B.W., ASHTON, M.S., DIELS, J., VAN DER MEER, B. and ENCLONA, E. Biomass estimations and carbon stock calculations in the oil palm plantations of African derived savannas using IKONOS data. International Journal of Remote Sensing, n. 25, p. 54475472, 2004.

TUCKER, C.J., VANPRAET, C.L., SHARMAN, M.J. e VAN ITTERSUM, G., Satellite remote sensing of total herbaceous biomass production in the Senegalese Sahel: 1980-1984. Remote Sensing of Environment, vol. 17, p. 233-249, 1985.

USGS GLOBAL VISUALIZATION VIEWER (USA). Frequently Asked Questions about the Landsat Missions. Disponível em: <http://landsat.usgs.gov/L8_band_combos.php>. Acesso em: Ago. 2018.

VERBESSELT, J., SOMERS, B., VAN AARDT, J., JONCKHEERE, I. e COPPIN, P., Monitoring herbaceous biomass and water content with SPOT VEGETATION time-series to improve fire risk assessment in savanna ecosystems. Remote Sensing of Environment, vol. 101, p. 399-414, 2006.

VIANA, H.; LOPES, D.; ARANHA, J. Predição de biomassa arbustiva lenhosa empregando dados do inventário e o índice de diferença normalizada extraído em imagens landsat5 TM. Revista Millenium, v. 14, n. 37, 2009.

WATZLAWICK, L. F.; KIRCHNER, F. F.; SANQUETTA, C. R. Estimativa de biomassa e carbono em floresta com Araucária utilizando imagens do satélite Ikonos II. Ciência Florestal, Santa Maria, vol. 19, n. 2, p. 169-181, 2009.

WYLIE, B.K., HARRINGTON, J.A., PRINCE, S.D. e DENDA, I., Satellite and ground-based pasture production assessment in Niger: 1986-1988. International Journal of Remote Sensing, vol. 12, p. 12811300, 1991.

WYLIE, B.K., DENDA, I., PIEPER, R.D., HARRINGTON JR., J.A., REED, B.C. e SOUTHWARD, G.M., Satellite-based herbaceous biomass estimates in the pastoral zone of Niger. Journal of Range Management, vol. 48, p. 159-164, 1995.

WESSELS, K.J., PRINCE, S.D., ZAMBATIS, N., MACFADYEN, S., FROST, P.E. e VAN ZYL, D., 
Relationship between herbaceous biomass and 1-km2 advanced very high resolu- tion radiometer (AVHRR) NDVI in Kruger National Park, South Africa. International Journal of Remote Sensing, vol. 27, p. 951-973, 2006.

WENDLING, J. L. G. Modelos matemáticos de crescimento e produção em biomassa para árvores de Euterpe edulis Mart. Plantadas a céu aberto. Dissertação (Mestrado em Ciências Florestais) - Setor de Ciências Agrárias, Universidade Federal do Paraná, Curitiba, 1998.

WOOLLEN, E.; RYAN, C. M.; BAUMERT, S.; VOLLMER, F.; GRUNDY, I.; FISHE, J.; FERNANDO, J.; LUZ, A.; RIBEIRO, N. e LISBOA, S.' N. Charcoal production in the Mopane woodlands of Mozambique: what are the trade-offs with other ecosystem services? Phil. Trans. R. Soc. B, v. 371, 2016. DOI: http://dx.doi.org/10.1098/rstb.2015.0315.

WONDRADE, N., DICK, OB; TVEITE., H. Estimating above Ground Biomass and Carbon Stock in the Lake Hawassa Watershed, Ethiopia by Integrating Remote Sensing and Allometric Equations, Forest Res. Vol. 4, n. 151. 2015.

\section{Biografia do autor principal}

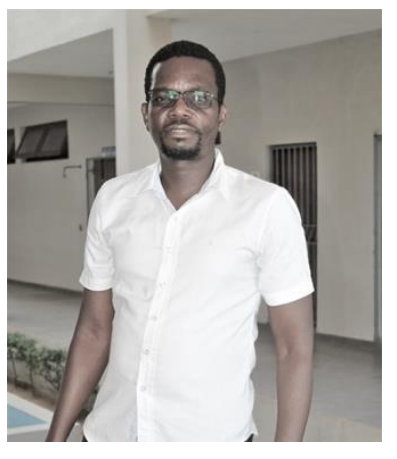

Idolgy Ribeiro dos Santos Mabunda, natural da cidade de Tete - Moçambique. Licenciado e Mestrado em Ensino de Geografia pela Universidade Pedagógica de Moçambique. Atualmente é doutorando em Energia e Meio ambiente pela Universidade Pedagógica estudando estimativa da biomassa lenhosa utilizando dados de sensoriamento. É professor na Universidade Save (Unisave), Faculdade de ciências naturais e exatas, atuando nas disciplinas de Cartografia, Geoprocessamento, Sistema de informação Geografia, Avaliação dos Recursos naturais. Seus interesses de pesquisa são no uso de sensoriamento remoto e sistema de informações geográficas para o monitoramento da vegetação, mudanças ambientais e na conservação da biodiversidade.

Esta obra está licenciada com uma Licença Creative Commons Atribuição 4.0 Internacional - CC BY.Esta licença permite que outros distribuam, remixem, adaptem e criem a partir do seu trabalho, mesmo para fins comerciais, desde que lhe atribuam o devido crédito pela criação original. 